\title{
Recovery from hybrid breakdown reveals a complex genetic architecture of mitonuclear incompatibilities
}

\author{
Ricardo Pereira ${ }^{1}$, Thiago Lima ${ }^{2}$, N Pierce ${ }^{3}$, Lin $\mathrm{Chao}^{4}$, and Ronald Burton ${ }^{3}$ \\ ${ }^{1}$ Ludwig Maximilians University Munich \\ ${ }^{2}$ University of California San Diego Scripps Institution of Oceanography \\ ${ }^{3}$ Scripps Institution of Oceanography \\ ${ }^{4}$ University of California San Diego Section of Ecology Behavior and Evolution
}

November 30, 2020

\begin{abstract}
Reproductive isolation is often achieved when genes that are neutral or beneficial in their genomic background become functionally incompatible in a foreign genome, causing inviability, sterility or low fitness in hybrids. Recent studies suggest that mitonuclear interactions are among the initial incompatibilities to evolve at early stages of population divergence across taxa. Yet, it is unclear whether mitonuclear incompatibilities involve few or many regions in the nuclear genome. We employ an experimental evolution approach starting with unfit F2 interpopulation hybrids of the copepod Tigriopus californicus, in which compatible and incompatible nuclear alleles compete in a fixed mitochondrial background. After about nine generations, we observe a generalized increase in population size and in survivorship, suggesting efficiency of selection against maladaptive phenotypes. Whole genome sequencing of evolved populations showed some consistent allele frequency changes across the three replicates of each reciprocal cross, but markedly different patterns between mitochondrial background. In only a few regions ( $6.5 \%$ of the genome), the same parental allele was overrepresented irrespective of the mitochondrial background. About $33 \%$ of the genome shows allele frequency changes consistent with divergent selection, with the location of these genomic regions strongly differing between mitochondrial backgrounds. The dominant allele matches the mitochondrial background in 87 and $89 \%$ of these genomic regions, consistent with mitonuclear coadaptation. These results suggest that mitonuclear incompatibilities have a complex polygenic architecture that differs between populations, potentially generating genome wide barriers to gene flow between closely related taxa.
\end{abstract}

\section{Introduction:}

Speciation occurs when populations accumulate genetic differences that cause reproductive incompatibilities in hybrids, leading to maladaptive phenotypes such as inviability, sterility, or to reduced fitness relative to parentals. Bateson (Bateson 1909), Dobzhansky (Dobzhansky 1937), and Muller (Muller 1942) have proposed that such incompatibilities would involve two or more loci, so that substitutions that are adaptive or neutral in their own genomic background can be functionally incompatible with alleles that are present in a foreign genomic background; the so-called Bateson-Dobzhansky-Muller incompatibilities or BDMIs. Although this model has guided research on the genomic basis of speciation for more than 70 years, only within the last decade have genomic regions (and in few cases genes) causing full sterility or inviability been identified in taxa as diverse as yeast, flies, mice and plants (Presgraves 2010). Such genes are found to evolve rapidly, as expected for genes caught up in open-ended molecular evolutionary arms race, and are often involved in co-evolution between host and pathogen, or between selfish genes and suppressors (Presgraves 2010). Particularly in studies with highly divergent species pairs, BDMIs seem to have a complex genomic architecture that involves tens of genes spread throughout the genome (Tang \& Presgraves 2009; Schumeret al. 2018). Yet, full sterility or inviability evolve later during the speciation process, and thus it is unclear 
whether those genes reflect initial barriers that arise between diverging populations or whether they reflect other evolutionary forces that arose after reproductive isolation was established. Therefore, understanding the genetic architecture of incompatibilities causing partial reproductive isolation, such as reduced fertility, remains an important task in evolutionary biology (Corbett-Detig et al. 2013; Turner \& Harr 2014; Rafati et al. 2018).

Recent studies in taxa as diverse as arthropods, nematodes, vertebrates, yeast, and angiosperms (reviewed in Burton et al. 2013; Sloanet al. 2017; Hill et al. 2018) have shown that the co-evolution between the mitochondrial and the nuclear genome often results in partially reduced fitness in hybrids between closely related taxa (i.e. species, subspecies, or even populations). This observation has led several authors to suggest that mitonuclear BDMIs play a disproportional role at early stages of reproductive isolation relative to the more widely studied nuclear-nuclear BDMIs (Burton \& Barreto 2012; Hill 2016). To understand why such a general pattern would arise across species, one needs to consider the origin and evolution of the mitochondrion in eukaryotes. Some 1.5 billion years ago, when a proteobacterial heterotroph (proto-mitochondrion; Martijn et al.2018) became the obligatory endosymbiont of an archaebacterial methanogen (proto-nucleus), this symbiosis resulted in a strong functional partition (Rivera et al. 1998), where the proto-mitochondrion became responsible for metabolic functions and the proto-nucleus for transcription and translation. Because intracellular clonal replication favors smaller genomes (Taylor et al. 2002; Clark et al. 2012), a race for replication of the proto-mitochondrion lead to the reduction in the endosymbiont genome size, both through the loss of redundant genes and through gene transfer to the host genome (Selosse et al. 2001; Timmis et al.2004). Such selective pressure is still observed today in the genome evolution of intracellular endosymbionts in insects (Wernegreen 2002), in the recent transposition of mitochondrial genes into the nuclear genome (Hazkani-Covo et al. 2010), or even in the deletion of essential mitochondrial genes with significant fitness costs for the organism (Taylor et al. 2002). Currently, in most metazoans, over 1,000 proteins are housed in the mitochondrion (Calvo \& Mootha 2010), but only 13 of those remain coded in the mitochondrial genome. Hence, mitochondrial function is dependent upon nuclear-encoded proteins, many of which interact closely with mtDNA encoded proteins, RNAs or DNA-binding sites. This mitonuclear cooperation sets the stage for a tight evolutionary arms race between the nuclear and mitochondrial genomes to preserve functionality within eukaryotic evolutionary lineages in essential cell functions, such as respiration and mitochondrial protein synthesis, that have large impacts on organismal fitness. Such mitonuclear coadaptation becomes exposed in F2 hybrids, where independently evolving mitochondrial and nuclear alleles are forced to interact, often being functionally incompatible, disrupting organelle function and, consequently, causing hybrid breakdown (Burtonet al. 2013).

Contrary to nuclear-nuclear BDMIs, mitonuclear incompatibilities are expected to be highly asymmetric for a variety reasons, as described in the model of compensatory coevolution (Rand et al. 2004). First, because of the mode of replication of its circular genome, mutation rate $(\mu)$ is higher in the mitochondrial relative to the nuclear genome. Second, due to its lack of recombination and matrilineal inheritance, mitochondrial genes have an effective population size $(\mathrm{Ne})$ that is 4 times smaller than that of nuclear genes, resulting in higher rates of fixation via genetic drift and conversely a reduced efficiency of selection. These two processes result in the consistent observation of faster evolution rates of mitochondrial relative to nuclear genes, which are 2-fold in drosophilids, 20-fold in ungulates and up to 40-fold in primates (Osada \& Akashi 2012). Even though most de novo mutations are partially deleterious, the relatively higher levels of genetic drift allow the fixation of these new mutations. This asymmetry of $\mu$ and $N e$ leads to a higher fixation rate for weakly deleterious mutations in the mitochondrial genomes relative to the nuclear genome; a process known as "Muller's ratchet" (Lynch \& Blanchard 1998). This accumulation of deleterious mutations in the mitochondrial genome elicits compensatory mutations in the interacting nuclear genes (Osada \& Akashi 2012; Barreto \& Burton 2013a; Sloan et al. 2014) but not in the rest of the nuclear genome, maintaining the stability and function of mitonuclear protein complexes. Although such mitochondrial deleterious mutations are effectively silenced within a population, they become exposed in interpopulation crosses where coadapted mitonuclear complexes become mismatched in hybrids, contributing for the establishment of genetic barriers between recently diverged taxa (Sloan et al. 2017). The magnitude of such early barriers to gene flow directly 
depends on the genetic architecture of mitonuclear BDMIs, which remains unknown across species.

Although mitonuclear incompatibilities are now recognized to play a general role in establishing reproductive isolation between emerging species (Reinhardt et al. 2013), their effect is most visible in taxa presenting exceptionally high mitochondrial evolution rates, such as the copepod Tigriopus californicus (Willett 2012). Allopatric divergence between populations of this species resulted in parallel patterns of genomic divergence that are consistent with mitonuclear coevolution happening within independent populations. Protein coding genes from the mitochondria evolve 2 to 14 times faster than those from the nuclear genome (Pereira et al. 2016), suggesting that mitochondrial genes drive intragenomic coevolution. Nuclear encoded proteins that functionally interact with the mitochondria evolve more rapidly than non-interacting nuclear encoded proteins (Barreto et al. 2018), suggesting that selection favoring compensatory mutations targets specific nuclear genes. Finally, experimental interpopulation F2 hybrids show that fitness breakdown in multiple life history traits (such as fecundity, survivorship and developmental time) scales with mitochondrial divergence (Burton 1990a; Edmands 1999). Notably, F2 fitness breakdown is rescued in maternal backcrosses (Ellison \& Burton 2008b), where mitochondrial and nuclear coevolving units are rematched, demonstrating that fitness breakdown is caused by interactions between the mitochondria and unknown nuclear loci. Despite such an evident parallelism in mitonuclear coevolution within populations, evolutionary processes conditioned by local habitat are non-parallel. For example, ecological trade-offs along latitudinal gradients have resulted in differential adaptation to temperature (Willett 2010; Hong \& Shurin 2015; Pereira et al. 2017) and to salinity (Leong et al.2017). Moreover, smaller $\mathrm{Ne}$ at southern populations has resulted in stronger genetic drift relative to northern populations (Pereiraet al. 2016), and potentially in a faster accumulation of deleterious mutations genome wide.

Recent studies with interpopulation hybrids of T. californicushave established that mitonuclear incompatibilities result in fitness breakdown at various organizational levels. While heterozygous F1s are vigorous, mismatched mitonuclear complexes in F2 and inbred lines results in: reduced mitochondrial function (Ellison \& Burton 2008a), reduced ATP production (Ellison \& Burton 2006), elevated oxidative damage to DNA (Barreto \& Burton 2013b), upregulation of pathways involved in physiologic stress (Barreto et al. 2015), and breakdown at multiple life history traits (fecundity, survivorship and developmental time; Burton 1990a; Edmands 1999). It is still unclear whether such generalized fitness breakdown cascade from mitonuclear BDMIs involving few nuclear genes with generalize effect, or involving multiple regions spread throughout the nuclear genome. It is worth noting that this species lacks sex chromosomes, and instead has polygenic sex determination (Voordouw \& Anholt 2002; Alexander et al. 2015). Therefore, factors leading to the disproportionate role of sex chromosomes in the evolution of BDMIs reported in many species (Presgraves 2018), does not apply in this case.

Previous efforts to understand the genetic architecture of mitonuclear BDMIs in T. californicus have focused on deviations from the expected Mendelian inheritance in F2 hybrids surviving to adulthood (Foley et al. 2013; Lima \& Willett 2018; Lima et al.2019), in hybrid swarms with recovered fitness (Pritchard \& Edmands 2012) or among high- and low-fitness hybrids within an F2 cohort (Healy \& Burton 2020). Although those generally confirm a prominent role of mitonuclear incompatibilities relative to nuclear-nuclear BDMIs (but see Pritchard et al. 2011), they strongly differ in the estimated number, location and effect size of nuclear loci interacting with the mitochondria (ranging from few loci to all 12 chromosomes). Understanding the genomic architecture of mitonuclear BDMIs requires a whole-genome approach, over multiple generations of hybridization and selection, and under divergent selection associated to alternative mitochondrial backgrounds. To address this, we employed an experimental evolution approach initiated with replicate populations of low-fitness F2 interpopulation hybrids. Over the course of the experiment, parental nuclear alleles competed in alternate mitochondrial backgrounds (i.e. treatment) established by reciprocal crosses, for about nine generations (Fig. 1). To assess the efficiency of natural selection in small experimental populations we tested for increase in population size and for fitness recovery in female fecundity and nauplii survivorship, which are known to be associated with mitonuclear incompatibilities. Next, to find regions likely responding to selection (uniform or divergent between treatments), we identified genomic regions with significant allelic frequency change during the experiment. Finally, to tease apart nuclear genomic regions 
involved in mitonuclear incompatibilities we identified regions that differ among alternative mitochondrial backgrounds. We found genomic regions likely involved in mitonuclear BDMIs on multiple chromosomes and that these regions differ between mitochondrial backgrounds, suggesting that mitonuclear incompatibilities have a complex and asymmetric genetic architecture.

\section{Material and Methods:}

\section{Sampling}

To understand the genetic architecture of mitonuclear incompatibilities we focused on two well-studied populations of Tigriopus californicus : San Diego (SD) and Santa Cruz (SC). These populations were suitable for a competition experiment between divergent nuclear alleles under a fixed mitochondrial background because previous studies have shown that: i) marked mitochondrial divergence (22.17\%; Pereira et al. 2016) has driven adaptive evolution targeting genes functionally interacting with the mitochondria (Barreto \& Burton 2013a), ii) $91 \%$ of the nuclear polymorphisms are fixed among parental populations (Pereira et al. 2016) allowing an accurate estimation of allelic frequencies in hybrids (Lima et al. 2019), iii) F2 hybrids show breakdown at all physiological and life history traits known to be associated with mitonuclear incompatibilities (Ellison \& Burton 2006; a; b; Barreto \& Burton 2013b; Barreto et al. 2015), and iv) strong selection during F2 development results in significant deviations from the expected Mendelian inheritance (Foley et al.2013; Healy \& Burton 2020).

Stock populations were collected in San Diego (32* 44' 41.17' N, 117* 15' 19.43" W) and Santa Cruz (36* 56' 58.32 " N, $122^{*} 2$ ' 48.98 " W). Multiple cultures from each site were maintained in $400 \mathrm{~mL}$ beakers at common garden at $20^{\circ} \mathrm{C}$ with a 12:12 L:D photoperiod, in filtered seawater mixed with ground "Algae Wafers" (Kyorin Co., Himeji, Japan). Beakers were monthly mixed to promote panmixia within populations and medium was renewed.

\section{Experimental evolution}

T. californicus is sexually dimorphic. An adult male clasps an immature female until her terminal molt, when she is then inseminated and released. Females can mate only once and use stored sperm to fertilize sequential clutches of eggs that can add to several hundred progeny (Vittor 1971). This species lacks heteromorphic sex chromosomes and recombination occurs only in males (Burton et al. 1981). We obtained virgin females by separating clasped pairs, and produced F1 with similar mixed nuclear genome and fixed mitochondrial backgrounds; i.e. SD mitochondrial background on SD x SC cross, and SC mitochondrial background on SC $\mathrm{x}$ SD. For each reciprocal cross, we outcrossed F1s to produce recombinant F2 hybrids that were allowed to mate randomly.

Each experimental line started with 100 outbred F2 gravid females. Lines evolved under these conditions for nine months with overlapping generations, replenishing the growing medium monthly. Since on average females reach adulthood in 2-3 weeks and produce multiple egg clutches until they are 4-6 weeks old, this experimental design corresponds to approximately nine generations of experimental evolution up to F11. This procedure was replicated 10 and 7 times for the SC and SD mitochondrial backgrounds, respectively. We followed the same procedure to generate one control line with fully matched nuclear and mitochondrial genomes for each parental population (i.e. SC x SC and SD x SD).

\section{Fitness recover y}

Relatively small experimental populations may lead to strong genetic drift, and conversely to limited response to selection imposed by the fixed mitochondrial backgrounds. If selection is strong relative to drift in evolved lines we expect an increase in productivity and associated recovery in one or multiple fitness traits associated with mitonuclear incompatibilities (Ellison \& Burton 2008b). To test these hypotheses, at the end of the experimental evolution, we measured: census size (as the number of adults after), fecundity (as the number of nauplii hatching from the first clutch of a female), and survivorship (as the fraction of nauplii surviving to 14 days). Fecundity was replicated between 4 to 12 times, depending on the number of available virgin females, and survivorship was replicated between 10 to 28 times, depending on the number of available gravid 
females. To monitor how average fitness varied along the course of the experiment, we have also measure survivorship 3 to 8 additional times, using 4 to 12 replicates. Additionally, we measure these two fitness traits for the initial reciprocal F2 hybrids and for the pure parental populations, as a reference for fitness breakdown and recovery respectively. We estimated mean plus +-1SE. We tested for significant hybrid breakdown by comparing fitness of the F2 hybrid with its maternal population, using a Mann-Whitney $U$ -test and an alpha of 0.05 in R 2.15.1 (R Development Core Team). We tested for significant recovery in lines for which the mean reached or passed the reference parental fitness, adjusting the P-value when multiple comparisons occur at the same time.

Allelic frequency change

To provide insights into the genetic architecture of mitonuclear incompatibilities we examined allelic frequency change across the whole genome in lines evolving under each mitochondrial background, relative to the initial F2 females. We used the pool-seq approach, which has recently been established for evolve-andresequencing studies (Schlotterer et al. 2015), including in T. californicus(Lima \& Willett 2018; Lima et al. 2019; Healy:2020fn; Griffithset al. 2020).

For lines evolving under each mitochondrial background, we selected three replicates that showed larger census size, no further decrease in fecundity, and some recovery in survivorship. We pooled 200 adult individuals, or the maximum available, and extracted genomic DNA using the phenol chloroform protocol and digestion with RNAse (Sambrook \& Russell 2001). For the initial F2 females, we also extracted DNA from pools of 100 adult outcross $\mathrm{F} 2$ females that gave rise to the experimental populations, after removing their egg sacs. All samples were sequenced in the Illumina HiSeq 2000 platform with 100 bp paired-end libraries. Reads were trimmed for quality using PoPoolation2 (Kofler et al. 2011), discarding bases with Phred quality scores lower than 25 and keeping reads of at least 50bp after trimming.

We only considered SNPs that were fixed between parental populations and therefore that can be used to determine the ancestry of the nuclear alleles favored in either mitochondrial background. We used the bioinformatics pipeline established by (Lima \& Willett 2018; Limaet al. 2019) (see references for details), and the syntenic reference genomes of SD and SC, where more than $90 \%$ of the genome is anchored to the 12 chromosomes (Barreto et al. 2018). In short, first, we made the two reference genomes equivalent in length and accuracy by adding N's to any position where either parental reference had an "N". Second, we established a list of fixed SNPs by i) performing reciprocal mapping of reads from one population (Barretoet al. 2018) against the reference of the other, ii) considering only "fixed" positions where all mapped reads showed an alternative nucleotide to the reference (coverage [?] 15), and iii) comparing reciprocal mapping to keep only SNPs that were "fixed" in both mappings. Third, we mapped our reads for each hybrid dataset to both parental references using BWA-MEM with default parameters (Li \& Durbin 2009) and keeping reads that mapped with MAPQ score $>20$. When mapping reads from hybrids to one of the parental references, there is a known bias towards an over-representation of reads of the reference population (Lima \& Willett 2018). By averaging the read counts for each SNP, from the alignment to each parental population, this reference-population bias is overcome. Allele counts for the fixed SD and SC alleles and respective frequencies were determined using PoPoolation2 (Kofler et al. 2011) (minimum coverage of minor allele [?] 4). Finally, data for all cross datasets were compared and only SNP that passed all filters up to this point, for both reciprocal crosses, were kept for statistical analysis of allele frequency change. These SNPs are expected to show high levels of variance, due to the variation on sequencing coverage and sampling alleles from the pool of individuals (Lima \& Willett 2018). We averaged allele counts (and frequencies) in non-overlapping windows of 500 consecutive SNPs. We refer to these as "genomic windows".

In each mitochondrial background separately, we determined which of these genomic windows show a significant allelic frequency change by comparing the initial F2 hybrids to the evolved hybrids, using the CochranMantel-Haenszel $(\mathrm{CMH})$ test as implemented in the mantelhaen.test function in $\mathrm{R}$ package ( $\mathrm{R}$ Development Core Team). This test operates on $2 \times 2$ contingency tables (times the number of replicates), comparing the counts for SD and SC alleles at the beginning and at end of the experiment, and thus it is highly sensitive to variation in sequencing coverage. To account for differences in coverage between the initial and evolved 
hybrids, we estimated initial counts of SD and SC alleles by multiplying the allelic frequency estimated in the initial F2 hybrid pool by the total coverage observed at each evolved hybrid separately (i.e. counts for $\mathrm{SD}+\mathrm{SC})$. This approach results in a P-value per genomic window, which reflects the probability of rejecting the null hypothesis of similar allelic frequency during experimental evolution in a given mitochondrial background.

We used a z-score of 2 to identify genomic windows showing higher $\mathrm{P}$-values relative to the average values observed genome wide, consistent with stronger selection driving allelic frequency change. We have estimated three z-score threshold based on three distributions of P-values: i) from both crosses taken together (corresponding to a $-\log 10$ (P-value) of 3.24), ii) only from crosses evolving under the SC mitochondria (corresponding to a $-\log 10$ (P-value) of 4.17), and iii) only from crosses evolving under the SD mitochondria (corresponding to a $-\log 10$ (P-value) of 1.73). Because the two higher thresholds were highly restrictive to identify windows under selection in lines with the SD mitochondrial background (only zero or five windows), we considered the lower threshold to compare windows across mitochondrial backgrounds. Since adjacent genomic windows are physically linked, we looked for consecutive genome windows that are under selection. We refer to these as genomic regions. Genomic regions were classified as under selection through the following criteria: i) at least 10 genomic windows with - $\log 10$ (P-value) ? 1; ii) with at least one window above a z-score of 2; and iii) ending with one window - $\log 10$ (P-value) ? 1. To represent which parental allele dominates during the experimental evolution, we classified genomic regions as blue for overrepresentation of the SC nuclear allele and red for overrepresentation of SD allele. By comparing allelic frequency change in reciprocal crosses, we identified genomic regions fitting three categories: "uniform selection", where the same allele dominates irrespective of the mitochondrial background; "divergent selection" where the dominant nuclear allele matches the mitochondria; and "antagonistic selection" where the dominant allele does not match the mitochondria. While we chose to use z-scores as threshold for selection, the results were qualitatively similar when considering a threshold based of the top $5 \%$ of the distribution of $\mathrm{P}$-values.

\section{Results:}

Fitness recovery

Both control parental lines went extinct after two generations of experimental evolution. In contrast, all hybrid lines survived the nine months of experimental evolution, with the exception of SC3, which went extinct in the 6th month. Most of the surviving lines showed an increase in population size (6/9 of the SC lines, and 6/7 of the SD lines), with census size showing an increase between 1.1-fold in SD4 to 9-fold in SD6 (Fig. 2A).

Regarding fecundity (Fig. 2B), F2 hybrids had lower fitness than the parental with the respective mitochondrial lineage, confirming F2 hybrid breakdown in this life history trait. Hybrid breakdown was more extreme in the comparison with the SC mitochondria $(\mathrm{P}$-value $=0.005)$, relative to that with the $\mathrm{SD}$ mitochondria $(\mathrm{P}$-value $=0.017)$, consistent with stronger breakdown in SC mitochondrial background. Of the hybrid lines that survived experimental evolution, $7 \mathrm{SC}$ and $6 \mathrm{SD}$ lines had sufficient virgin females to assess fecundity. Mean fecundity +-1SE of all hybrid lines was within the range observed in the respective F2 hybrids (mean plus +-1SE), consistent with no or little recovery from initial hybrid breakdown.

Regarding survivorship (Fig. 2C), both reciprocal hybrids had lower fitness than parental populations with the respective mitochondrial lineage, yet non-significant ( $\mathrm{P}$-values $>0.125$ ), confirming breakdown in this trait. The +-1SE intervals overlapped in SD but not in the SC comparison, again suggesting that F2 hybrid breakdown is stronger in the SC mitochondrial background. A total of $8 \mathrm{SC}$ and $6 \mathrm{SD}$ lines had sufficient gravid females to assess survivorship of nauplii. Of these, 4 SC lines and 4 SD lines had survivorship (mean +1SE) at or above the fitness of the respective parental, suggesting recovery from the initial hybrid breakdown. These differences were significant for the SC4, SC7, SC8, SD3 and SD7 lines (respective corrected P-values= 0.001, 0.009, 0.017, 0.043, 0.043; Fig. 2C).

Based on fewer replicates measured during the experiment, we observed a large temporal variability on survivorship (Fig. 2D, Fig. S1). The increase of survivorship was more noticeable among SC lines, where 
F2 breakdown was stronger, and lines stayed for a few generations at the fitness level of their parental population.

Based on these results, for the lines evolving under the SC mitochondria, we chose to sequence SC4, SC8 and SC10 because, from lines with the highest increase of population size, these were the ones with highest survivorship at the end of the experiment. For the lines evolving under the SD mitochondria, we chose to sequence SD3, SD4, and SD6 because they showed some increase in survivorship, without a noticeable decrease in fecundity (contrary to SD7). We note that, although the choice of lines is necessarily subjective, given that this experimental design did not employ direct selection on a specific phenotype, surviving lines showing population growth would be good representatives of recovery from initial F2 breakdown.

Allelic frequency change

We sequenced between 53 and 74 million reads per pool (BioProject XXXX). After quality trimming and mapping, this sequencing effort resulted in an average coverage of 31.9x (SC mtDNA) and 41.1x (SD mtDNA) for the initial F2 hybrids, 33.9x, 43.1x and 35.4x for the lines evolving under the SC mitochondria, and 33.5x, $33.5 \mathrm{x}$ and $34.5 \mathrm{x}$ for the lines evolving under the SD mitochondria. After reciprocal mapping to the reference genomes of both parental populations and filtering for fixed SNPs, our approach allowed us to estimate read counts for 1,658,000 ancestry informative SNPs, corresponding to 3,316 genomic windows, and covering the 12 chromosomes of $T$. californicus .

The allelic frequencies in adult F2 were generally close to $50 \%$ (Figure S2), varying between 38 and $64 \%$ in the F2s with the SC mitochondria, and between 34 and $61 \%$ in the F2s with the SD mitochondria. This variation was relatively stable along each of the 12 chromosomes, consistent with chromosome-wide linkage disequilibrium. In contrast, allelic frequencies in hybrid lines after experimental evolution showed a strong deviation from 50\% (Figure S3). This deviation was most pronounced in the lines evolving under the SC mitochondrial background, where the frequency of the SD allele ranged between 5 and $90 \%$. The lines evolving under the SD mitochondrial background showed a more modest allele frequency change, ranging from 20 and $76 \%$ for the SD allele. Contrary to F2s, changes in allelic frequency were variable along each of the 12 chromosomes, showing that the effective population recombination rate was enough to reduce linkage disequilibria during the nine months of experimental evolution.

The SC lines showed the most extreme P-values of rejecting the null hypothesis of no allelic frequency change, with genomic windows reaching the threshold of $-\log 10$ (P-value) $=1.73$ in all 12 chromosomes. Larger genomic regions were identified in 8 chromosomes, 7 of which favored the nuclear allele matching the mitochondrial background (i.e. SC allele). The only region in which the opposite allele was favored (i.e. SD allele in chromosome 11) did not show a similar pattern in lines evolving under the SD mitochondria. The SD lines showed less extreme P-values supporting allelic frequency change, with genomic windows above the threshold located in 6 chromosomes. Larger genomic regions were identified in 5 chromosomes, 3 of which favored the nuclear allele matching the mitochondrial background (i.e. SD allele). Notably, the chromosomal regions in which the opposite parental allele was favored (i.e. SC allele in chromosomes 7 and 8), show a similar pattern in the SC lines. Genomic regions responding to selection varied between $62 \mathrm{Kbp}-6.2 \mathrm{Mbp}$ for the SC lines and $512 \mathrm{Kbp}-3.2 \mathrm{Mbp}$ for the SD lines.

From the 3,316 genomic windows analyzed here, $60.5 \%$ were not skewed in both lines and occurred in all 12 chromosomes, consistent with a prevalent role of genetic drift. About $6.5 \%$ of the windows shows a consistent skew towards the SC allele, mostly in chromosome 7 and in part of chromosome 8, consistent with uniform selection favoring SC alleles. The SD alleles were never consistently favored in both mitochondrial backgrounds. The remaining $33 \%$ of the genome show skews dependent on the mitochondrial background and are therefore consistent with divergent selection.

Of the 909 genomic windows that are skewed only in the SC mitochondrial background, $88.6 \%$ show a skew favoring the matching SC nuclear allele, in chromosomes 2, 3, 8,10 and 12. The remaining $11.4 \%$, all in chromosome 11, show a skew towards the mismatched SD nuclear allele. Of the 187 genomic windows that are skewed only in the SD mitochondrial background, $87 \%$ show a skew towards the matching SD nuclear 
allele, in chromosomes 4, 6, and 9. The remaining $13 \%$ show a skew towards the mismatching SC nuclear allele, but notably all these windows are adjacent to windows likely under uniform selection favoring the same allele in chromosome 7 .

\section{Discussion:}

A mounting body of work has suggested that mitonuclear incompatibilities are among the first genetic barriers to evolve at early stages of population divergence across taxa (Burton \& Barreto 2012; Burtonet al. 2013; Hill 2016; Sloan et al. 2017; 2018; Hillet al. 2018), affecting multiple life history traits that render hybrids unfit. Nevertheless, it is still unclear whether such BDMIs involve a few nuclear regions in a localized part of the genome, or if they involve many regions throughout the nuclear genome. Understanding the genetic architecture of mitonuclear incompatibilities can benefit from the "evolve-and-resequence" approach developed for model organisms (Burke et al. 2014; Schlotterer et al. 2015), where adaptation to divergent environments stems from standing genetic variation generated from an initial hybrid population. In this study, we evolved sickly F2 hybrids of the copepod Tigriopus californicus, where parental nuclear alleles are represented at similar frequencies, and monitor change in these allele frequencies after experimental evolution under two divergent mitochondrial backgrounds. We hypothesize that recovery of hybrid fitness will primarily result from selection favoring mitonuclear compatibility and that allelic frequency changes across the genome will reveal locations where such selection has acted.

Increased productivity in hybrid lines suggests recovery from fitness breakdown

Previous long-term evolution experiments in T. californicus have found varying degrees of fitness recovery from F2 breakdown (Hwanget al. 2011; Pritchard et al. 2012; Hwang et al.2016), presumably because hybrids with matching mitonuclear complexes would have higher generalized fitness than their siblings with unmatched mitonuclear complexes. Thus, matched (coadapted) nuclear alleles would outcompete unmatched (incompatible) alleles over multiple generations (Fig. 1). However, those experimental lines started with a mix of pure parental individuals, so that unmixed nuclear genotypes and the two mitochondria could persist well into the experiment. This design made it challenging to distinguish between fitness recovery caused by the exclusion of incompatible nuclear alleles in a hybrid background, from fitness recovery caused by competition and drift of pure parental genotypes. In contrast, we initiated experimental lines with F2 hybrids and in fixed mitochondrial backgrounds, so that differences in fitness and allele frequency can only be explained by competition between parental nuclear alleles.

Our results show that control parental populations went extinct within two months of experimental evolution, probably due to inbreeding depression described in this species (Palmer \& Edmands 2000). In contrast, almost all hybrid lines survived the nine months of experimental evolution, with most showing an increase in population size (from 1.1 to 9-fold; Fig. 2A). From the many traits known to be involved in hybrid breakdown in T. californicus (Burton 1990a; b; Edmands 1999; Edmands \& Burton 1999; Ellison \& Burton 2006; 2008a; 2010), we have assessed recovery in two: female fecundity and nauplii survivorship. In respect to fecundity, we observed a strong F2 breakdown (P-values $<0.017$ ), and no recovery in evolved hybrid lines, irrespective of the mitochondrial background (Fig. 2B). This suggests that this is a complex trait that could not evolve in these experimental conditions of population size, number of generations and selective regime.

In contrast, with respect to survivorship, we observed less severe breakdown in F2 generation (P-values> 0.125). Notably, survivorship breakdown was stronger in the F2 hybrids with the SC mitochondria, suggesting that selection imposed by mitonuclear incompatibilities is stronger in SC relative to SD mitochondrial background. In both backgrounds, several hybrid lines recovered up to or beyond parental fitness levels (P-values $<0.043$; Fig. 2C), suggesting that recombination and selection over nine months were sufficient to cause evolution at this trait. Rescue in survivorship up to or beyond parental fitness levels has also been reported in F9 recombinant hybrid lines of T. californicus (Pereira et al. 2014), suggesting that the genetic architecture of this specific trait might be relatively simple. Nevertheless, we note that the line showing the largest increase in productivity (9-fold increase in SD6), did not show recovery in survivorship, therefore selection imposed by our experimental design is likely multifarious and responding with phenotypes beyond 
those measured here. Future studies employing directed selection on a specific trait (Healy \& Burton 2020) over many generations of experimental evolution may provide insights into this question.

Allele frequency change is consistent with asymmetric mitonuclear incompatibilities

By measuring allelic frequency change from the initial F2 hybrids to the replicated hybrid lines that evolved under each mitochondrial background, we can scan the genome for regions likely under selection, which should be consistent across replicates, from regions under genetic drift, which should show no or inconsistent change across replicates. Although the choice of sequenced lines is debatable, we assume that the three chosen replicates were adequate for testing our hypothesis because they all show increased in population size, and survivorship has recovered to parental fitness for multiple generations (Fig. 2D). In agreement with selection, we found some consistency among replicate populations of each reciprocal cross (Fig. S2). This was particularly pronounced on the SC lines, where we found more extreme P-values rejecting the null hypothesis of genetic drift (Fig. 3); also reflected by the higher lineage-specific thresholds based on a z-score of 2 (- $\log 10$ (P-value) of 4.17 for SC and 1.73 for SD). Together with our observation that F2 breakdown in survivorship is stronger in the SC background (Fig. 2C), this suggests that the strength of selection acting against mitonuclear incompatibilities was stronger in this mitochondrial background relative to SD. Such asymmetries in the strength of mitonuclear incompatibilities is also supported by reciprocal crosses of multiple populations of T. californicus (Ganz \& Burton 1995; Peterson et al. 2013).

By comparing the genomic regions responding to selection in lines evolving under alternative mitochondrial backgrounds, we can distinguish regions responding to uniform selection (i.e. where the same nuclear allele dominates), from regions responding to divergent selection (i.e. where different alleles dominate). Consistent with uniform selection in $6.5 \%$ of the genome (Table 1), we find that the SC allele dominates in much of chromosome 7 and a small segment of chromosome 8. Uniform selection could be explained by BDMIs between 2 or more nuclear factors (nuclear-nuclear instead of mitonuclear incompatibilities), purging of slightly deleterious alleles that have been fixed in one of the parental populations, or by adaptation to laboratorial conditions favoring alleles of one of the populations. These possibilities are also not mutually exclusive, and previous work in this species lend support to any of these. Studies with reciprocal F2 hybrids from multiple population crosses have found skews towards the same allele in one or two chromosomes (Foley et al. 2013; Lima et al. 2019), suggesting a modest role of nuclear-nuclear incompatibilities in T. californicus . Previous genomic studies of natural populations found that effective population size in SD is less than half that of SC (Pereira et al. 2016), and thus this population is expected to accumulate deleterious mutations twice as fast. Moreover, experimental studies have revealed that these populations are differentially adapted to temperature (Willett 2010; Hong \& Shurin 2015; Pereira et al.2017) and to salinity (Leong et al. 2017), and that rearing temperature used in this study favors SC alleles, relative to SD (Willett 2010). While these three selective regimes are impossible to distinguish under our experimental design, future evolveand-resequencing experiments varying environmental factors (such as in Griffiths et al. (2020), but with reciprocal mitochondrial backgrounds) will shed light on the relative role of ecological adaptation driving this signature of uniform selection.

Consistent with divergent selection, we find that allele frequencies differ in $33 \%$ of the genome, depending on the mitochondrial background. Yet, concerning the targets of divergent selection, there is no region where opposite alleles dominate, as would be expected if the same genomic region was under divergent selection in both mitochondrial backgrounds, i.e. if mitonuclear incompatibilities had a symmetric genetic architecture. Instead, the location of genomic regions consistent with divergent selection strongly differs among mitochondrial backgrounds. For example, the SC mitochondria seems to interact strongly with regions in chromosomes $2,3,8,9,11$ and 12 , while the SD mitochondria seems to interact with regions in chromosomes 4, 6 and 9 (Fig. 3). In agreement, previous studies using F2 hybrids from reciprocal crosses have reported allele frequency changes during a single generation in multiple chromosomes, both in response selection for rapid development ( 5 chromosomes, in Healy \& Burton 2020) or simply as a consequence of differential survivorship during development (4 to 5 chromosomes, in Lima et al (2019)). In contrast to those studies, where skews consistent with divergent selection were small in magnitude (up to $15 \%$ ) and 
were consistent across entire chromosomes, by evolving hybrids over multiple generations, we were able to identify genomic regions that span between half to small portions of the chromosomes $(62 \mathrm{Kbp}-6.2 \mathrm{Mbp}$; Fig. 3). Nevertheless, our regions under divergent selection still contain hundreds or thousands of genes, and thus prevent identifying the causal genes underlying mitonuclear incompatibilities. Future "evolve-andresequence" studies increasing effective recombination rate, either by increasing the size of experimental populations or the number of generations under experimental evolution, will provide higher resolution into the asymmetric and polygenic genomic architecture of mitonuclear incompatibilities described here.

Consistent with our hypothesis, the direction of divergent selection favoring mitonuclear adaptation is strongly supported, as nuclear alleles matched the mitochondrial background in 88.6 and $87 \%$ of the cases (Table 1). Some of the remaining windows with skews towards the mismatched allele can be explained by non-biological reasons. For example, in SD lines, all windows showing skews towards the mismatched SC allele are physical linked to a large region of chromosome 7 where the SC allele is favored in both mitochondrial backgrounds, and thus can be explained by the stochasticity of recombination events during this experiment. In contrast, in SC lines, windows with the mismatched allele can only be explained by antagonistic selection, as these are located in half of chromosome 11, which shows no change in the reciprocal cross. Although this pattern is usually not expected for mitonuclear incompatibilities, empirical studies in F2 hybrids show that it is not uncommon. In studies with F2 hybrids, Lima et al 2019 found patterns consistent with antagonistic selection in one to three chromosomes, depending on the population cross, and Healy \& Burton (2020) found such pattern in F2 hybrids between SD and SC also in chromosome 11.

Taken together, our results offer new insights into the genomic architecture of mitonuclear incompatibilities. We find that mitonuclear incompatibilities are highly asymmetric, both in the magnitude of selection and in the location of targeted nuclear regions, supporting theoretical predictions of BDMIs involving uniparentally inherited genes (Turelli \& Moyle 2007). Despite the small size and limited gene content of the mitochondrial genome, we find that it exerts significant divergent selection in many genomic regions located in multiples chromosomes of the nuclear genome. Our finding that mitonuclear interactions predominantly favor coadapted gene complexes imply that such early BDMIs can result in barriers to gene flow that are widespread across the genome. The rapid evolution of the mitochondria and coevolving nuclear genes during periods of geographic isolation, may thus constitute important reproductive barriers that persist after taxa establish secondary contact or sympatry.

\section{Acknowledgements:}

This study was supported by grants from the U.S. National Science Foundation (DEB1556466 and IOS1754347 to RSB). RJP was supported by the UCSD Frontiers of Innovation Scholars Program, and TGL was supported by the NSF PRFB Award no. 1523543. We thank M. Burke for the helpful discussions setting up this experiment and preparing the analyses of the genomic data.

\section{References:}

Alexander HJ, Richardson JML, Edmands S, Anholt BR (2015) Sex without sex chromosomes: genetic architecture of multiple loci independently segregating to determine sex ratios in the copepod Tigriopus californicus . Journal of Evolutionary Biology , 28 , 2196-2207.

Barreto FS, Burton RS (2013a) Evidence for Compensatory Evolution of Ribosomal Proteins in Response to Rapid Divergence of Mitochondrial rRNA. Molecular Biology and Evolution , 30 , 310-314.

Barreto FS, Burton RS (2013b) Elevated oxidative damage is correlated with reduced fitness in interpopulation hybrids of a marine copepod.Proceedings Of The Royal Society B-Biological Sciences ,280 , 2013152120131521.

Barreto FS, Pereira RJ, Burton RS (2015) Hybrid Dysfunction and Physiological Compensation in Gene Expression. Molecular Biology and Evolution, 32, 613-622.

Barreto FS, Watson ET, Lima TG et al. (2018) Genomic signatures of mitonuclear coevolution across 
populations of Tigriopus californicus . Nature Publishing Group , 2 , 1250-1257.

Burke MK, Liti G, Long AD (2014) Standing Genetic Variation Drives Repeatable Experimental Evolution in Outcrossing Populations of Saccharomyces cerevisiae. Molecular Biology and Evolution ,31 , 3228-3239.

Burton RS (1990a) Hybrid breakdown in developmental time in the copepod Tigriopus californicus . 18141822 .

Burton RS (1990b) Hybrid Breakdown in Physiological-Response - a Mechanistic Approach. 44, $1806-1813$.

Burton RS, Barreto FS (2012) A disproportionate role for mtDNA in Dobzhansky-Muller incompatibilities? Molecular Ecology ,21 , 4942-4957.

Burton RS, Feldman MW, Swisher SG (1981) Linkage relationships among five enzyme-coding gene loci in the copepod Tigriopus californicus : a genetic confirmation of achiasmiatic meiosis.Biochem. Genet. , 19 , $1237-1245$.

Burton RS, Pereira RJ, Barreto FS (2013) Cytonuclear Genomic Interactions and Hybrid Breakdown. 44 , $281-302$.

Calvo SE, Mootha VK (2010) The Mitochondrial Proteome and Human Disease.Annual review of genomics and human genetics , 11 , 25-44.

Clark KA, Howe DK, Gafner K et al. (2012) Selfish little circles: transmission bias and evolution of large deletion-bearing mitochondrial DNA in Caenorhabditis briggsae nematodes. (C Braendle, Ed,). PLoS ONE , 7, e41433.

Corbett-Detig RB, Zhou J, Clark AG, Hartl DL, Ayroles JF (2013) Genetic incompatibilities are widespread within species. Nature ,504, 135-137.

Dobzhansky T (1937) Genetics and the origin of species . New York.

Edmands S (1999) Heterosis and outbreeding depression in interpopulation crosses spanning a wide range of divergence. 53 , 1757-1768.

Edmands S, Burton R (1999) Cytochrome C oxidase activity in interpopulation hybrids of a marine copepod: A test for nuclear-nuclear or nuclear-cytoplasmic coadaptation. 53 , 1972-1978.

Ellison CK, Burton RS (2006) Disruption of mitochondrial function in interpopulation hybrids of Tigriopus californicus . 60 , 1382-1391.

Ellison CK, Burton RS (2008a) Genotype-dependent variation of mitochondrial transcriptional profiles in interpopulation hybrids.Proceedings of the National Academy of Sciences , 105 , 15831.

Ellison CK, Burton RS (2008b) Interpopulation hybrid breakdown maps to the mitochondrial genome. $\mathbf{6 2}$, 631-638.

Ellison CK, Burton RS (2010) Cytonuclear conflict in interpopulation hybrids: the role of RNA polymerase in mtDNA transcription and replication. Journal of Evolutionary Biology , 23 , 528-538.

Foley BR, Rose CG, Rundle DE, Leong W, Edmands S (2013) Postzygotic isolation involves strong mitochondrial and sex-specific effects in Tigriopus californicus, a species lacking heteromorphic sex chromosomes. Heredity .

Ganz HH, Burton RS (1995) Genetic differentiation and reproductive incompatibility among Baja California populations of the copepod Tigriopus californicus . Marine Biology , 123 , 821-827.

Griffiths JS, Kawji Y, Kelly MW (2020) An experimental test of adaptive introgression in locally adapted populations of splashpool copepods. Molecular Biology and Evolution. 
Hazkani-Covo E, Zeller RM, Martin W (2010) Molecular Poltergeists: Mitochondrial DNA Copies (numts) in Sequenced Nuclear Genomes (HS Malik, Ed,). PLoS Genetics, 6 , e1000834-11.

Healy TM, Burton RS (2020) Strong selective effects of mitochondrial DNA on the nuclear genome. Proceedings of the National Academy of Sciences , 117 , 6616-6621.

Hill GE (2016) Mitonuclear coevolution as the genesis of speciation and the mitochondrial DNA barcode gap. Ecology and Evolution ,6 , 5831-5842.

Hill GE, Havird JC, Sloan DB et al. (2018) Assessing the fitness consequences of mitonuclear interactions in natural populations.Biol. Rev. Camb. Philos. Soc. , 94, 1089-1104.

Hong BC, Shurin JB (2015) Latitudinal variation in the response of tidepool copepods to mean and daily range in temperature.Ecology, $96,2348-2359$.

Hwang AS, Northrup SL, Alexander JK, Vo KT, Edmands S (2011) Long-term experimental hybrid swarms between moderately incompatibleTigriopus californicus populations: hybrid inferiority in early generations yields to hybrid superiority in later generations. Conservation Genetics , 12 , 895-909.

Hwang AS, Pritchard VL, Edmands S (2016) Recovery from hybrid breakdown in a marine invertebrate is faster, stronger and more repeatable under environmental stress. Journal of Evolutionary Biology , 1-33.

Kofler R, Pandey RV, Schlotterer C (2011) PoPoolation2: identifying differentiation between populations using sequencing of pooled DNA samples (Pool-Seq). Bioinformatics , 27 , 3435-3436.

Leong W, Sun PY, Edmands S (2017) Latitudinal Clines in Temperature and Salinity Tolerance in Tidepool Copepods. The Journal of heredity, 109 , 71-77.

Li H, Durbin R (2009) Fast and accurate short read alignment with Burrows-Wheeler transform. Bioinformatics , 25, 1754-1760.

Lima TG, Willett CS (2018) Using Pool-seq to Search for Genomic Regions Affected by Hybrid Inviability in the copepod T. californicus .The Journal of heredity , 109, 469-476.

Lima TG, Burton RS, Willett CS (2019) Genomic scans reveal multiple mito-nuclear incompatibilities in population crosses of the copepod Tigriopus californicus ., 73, 609-620.

Lynch M, Blanchard JL (1998) Deleterious mutation accumulation in organelle genomes. Genetica, 102-103 , 29-39.

Martijn J, Vosseberg J, Guy L, Offre P, Ettema TJG (2018) Deep mitochondrial origin outside the sampled alphaproteobacteria.Nature , 557 , 101-105.

Muller HJ (1942) Isolating mechanisms, evolution and temperature.Biological Symposia , 6 , 71-125.

Osada N, Akashi H (2012) Mitochondrial-nuclear interactions and accelerated compensatory evolution: evidence from the primate cytochrome C oxidase complex. Molecular Biology and Evolution , 29 , 337-346.

Palmer CA, Edmands S (2000) Mate choice in the face of both inbreeding and outbreeding depression in the intertidal copepod Tigriopus californicus . Marine Biology , 136 , 693-698.

Pereira RJ, Barreto FS, Burton RS (2014) Ecological novelty by hybridization: experimental evidence for increased thermal tolerance by transgressive segregation in Tigriopus californicus .,68 , 204-215.

Pereira RJ, Barreto FS, Pierce NT, Carneiro M, Burton RS (2016) Transcriptome-wide patterns of divergence during allopatric evolution.Molecular Ecology , 25 , 1478-1493.

Pereira RJ, Sasaki MC, Burton RS (2017) Adaptation to a latitudinal thermal gradient within a widespread copepod species: the contributions of genetic divergence and phenotypic plasticity. Proceedings Of The Royal Society B-Biological Sciences, 284, 20170236-8. 
Peterson DL, Kubow KB, Connolly MJ et al. (2013) Reproductive and phylogenetic divergence of tidepool copepod populations across a narrow geographical boundary in Baja California. Journal of Biogeography , $40,1664-1675$.

Presgraves DC (2010) The molecular evolutionary basis of species formation. Nature Reviews Genetics , 11 , $175-180$.

Presgraves DC (2018) Evaluating genomic signatures of "the large X-effect" during complex speciation. Molecular Ecology ,27, 3822-3830.

Pritchard VL, Edmands S (2012) The genomic trajectory of hybrid swarms: outcomes of repeated crosses between populations of Tigriopus californicus . Evolution 67, 774-791.

Pritchard VL, Dimond L, Harrison JS et al. (2011) Interpopulation hybridization results in widespread viability selection across the genome in Tigriopus californicus . BMC genetics ,12, 54 .

Pritchard VL, Knutson VL, Lee M, Zieba J, Edmands S (2012) Fitness and morphological outcomes of many generations of hybridization in the copepod Tigriopus californicus . Journal of Evolutionary Biology , 26 , 416-433.

Rafati N, Blanco-Aguiar JA, Rubin CJ et al. (2018) A genomic map of clinal variation across the European rabbit hybrid zone.Molecular Ecology , 27 , 1457-1478.

Rand DM, Haney RA, Fry AJ (2004) Cytonuclear coevolution: the genomics of cooperation. TREE , 19 , 645-653.

Reinhardt K, Dowling DK, Morrow EH (2013) Medicine. Mitochondrial replacement, evolution, and the clinic. Science, $341,1345-1346$.

Rivera MC, Jain R, Moore JE, Lake JA (1998) Genomic evidence for two functionally distinct gene classes. Proceedings of the National Academy of Sciences of the United States of America, 95 ,6239-6244.

Sambrook J, Russell DW (2001) Molecular Cloning . CSHL Press.

Schlotterer C, Kofler R, Versace E, Tobler R, Franssen SU (2015) Combining experimental evolution with next-generation sequencing: a powerful tool to study adaptation from standing genetic variation.Heredity , $114,431-440$.

Schumer M, Xu C, Powell DL et al. (2018) Natural selection interacts with recombination to shape the evolution of hybrid genomes.Science, 360, 656-660.

Selosse MA, Albert B, Godelle B (2001) Reducing the genome size of organelles favours gene transfer to the nucleus. Trends in Ecology \& Evolution, 16 , 135-141.

Sloan DB, Havird JC, Sharbrough J (2017) The on-again, off-again relationship between mitochondrial genomes and species boundaries. Molecular Ecology , 25 , 2325-25.

Sloan DB, Triant DA, Wu M, Taylor DR (2014) Cytonuclear Interactions and Relaxed Selection Accelerate Sequence Evolution in Organelle Ribosomes.Molecular Biology and Evolution , 31 ,673-682.

Sloan DB, Warren JM, Williams AM et al. (2018) Cytonuclear integration and co-evolution. Nature Reviews Genetics , 1-14.

Tang S, Presgraves DC (2009) Evolution of the Drosophila nuclear pore complex results in multiple hybrid incompatibilities. Science, 323, 779-782.

Taylor DR, Zeyl C, Cooke E (2002) Conflicting levels of selection in the accumulation of mitochondrial defects in Saccharomyces cerevisiae.Proceedings of the National Academy of Sciences of the United States of America , 99 , 3690-3694. 
Timmis JN, Ayliffe MA, Huang CY, Martin W (2004) Endosymbiotic gene transfer: organelle genomes forge eukaryotic chromosomes. Nature Reviews Genetics , 5 , 123-135.

Turelli M, Moyle LC (2007) Asymmetric postmating isolation: Darwin's corollary to Haldane"s rule. Genetics , 176 , 1059-1088.

Turner LM, Harr B (2014) Genome-wide mapping in a house mouse hybrid zone reveals hybrid sterility loci and Dobzhansky-Muller interactions. Elife , 3 , 4803-25.

Vittor BA (1971) Effects of the environment on fitness-related life history characters in Tigriopus californicus

Voordouw MJ, Anholt BR (2002) Environmental sex determination in a splash pool copepod. Biological Journal Of The Linnean Society ,76 , 511-520.

Wernegreen JJ (2002) Genome evolution in bacterial endosymbionts of insects. Nature Reviews Genetics , 3, 850-861.

Willett CS (2010) Potential fitness trade-offs for thermal tolerance in the intertidal copepod Tigriopus californicus . $64,2521-2534$.

Willett CS (2012) Quantifying the Elevation of Mitochondrial DNA Evolutionary Substitution Rates Over Nuclear Rates in the Intertidal Copepod Tigriopus californicus . Journal of Molecular Evolution , 74, $310-318$.

\section{Data Accessibility:}

Raw reads were deposited in the NCBI Sequence Read Archive (BioProject XXXX; BioSample Acc. XXXX). The dryad archive (doi: XXXX) contains the reference genomes, estimated allelic frequencies, P-values for allelic frequency change, list of genomic windows under selection, and fitness data.

\section{Author Contributions:}

RJP conceived the project with direct input from LC and RSB. RJP performed the research. RJP, TGL and NTP analyzed the data. RJP, TGL, and RSB wrote the manuscript, and all authors approved the final version.

Tables:

Table 1. Comparison of allelic frequency change in the genome of T. californicus, after 9 months of experimental evolution under divergent mitochondrial backgrounds.

Consistent with genetic drift:

Consistent with uniform selection:

Consistent with directional selection in both lines (symmetric selection):

Consistent with directional selection only in SC (asymmetric selection):

Consistent with directional selection only in SD (asymmetric selection):
Consistent with genetic drift:

no skew in both

Consistent with uniform selection:

$\mathrm{SC}$ allele is favored in both

$\mathrm{SD}$ allele is favored in both

Consistent with directional selection in both line matched allele in both

mismatched allele in both

Consistent with directional selection only in SC

$\mathrm{SC}$ allele is favored in SC (matched)

$\mathrm{SD}$ allele is favored in $\mathrm{SC}$ (mismatched)

Consistent with directional selection only in SD

$\mathrm{SD}$ allele is favored in SD (matched)

$\mathrm{SC}$ allele is favored in SD (mismatched) 


\section{SD- San Diego; SC- Santa Cruz}

\section{Figures:}

Figure 1. Competition experiment to reveal genomic regions involved in mitonuclear incompatibilities and their genomic architecture. Experimental hybridization results in F1 hybrids with the same nuclear composition but fixed alternative mitochondrial backgrounds (SD in red and SC in blue). Experimental evolution starts with F2 hybrids where recombination generated matched and mismatched mitonuclear complexes, leading to a decrease in average fitness of the starting population. Variation of individual fitness at multiple life history traits favors matched mitonuclear genotypes, leading to a recovery of life history traits and census size of the evolved populations. Comparison of allelic frequency change between lines evolving under alternative mitochondrial backgrounds allows distinguishing between genomic regions affected by uniform selection (e.g. adaptation, purging of deleterious mutations or nuclear-nuclear incompatibilities) from those affected by divergent selection related to the mitochondria (i.e. symmetric and asymmetric mitonuclear incompatibilities). In a fourth less likely scenario (not pictured here) a nuclear allele is favored in a mismatched mitochondrial background.

Figure 2. Variation of proxies for fitness during experimental evolution of hybrid lines under two alternative mitochondrial backgrounds (SC in blue, and SD in red). A. Population size expressed as the number of adult individuals. B. Female fecundity as the number of nauplii in the first clutch. C.Nauplii survivorship during 14 days of development. D. Temporal change in nauplii survivorship in the lines selected for sequencing (see Fig. S1 for other lines). Dark grey bars and rectangles refers to initial F2 hybrids (mean $\pm 1 \mathrm{SE}$ ), and light grey refers to the reference parental population. Lines selected for sequencing are marked in darker colors. Significant differences $(\mathrm{P}$-values $<0.05)$ are marked with *.

Figure 3. Consistent allele frequency changes identify genomic regions involved in mitonuclear incompatibilities. Genomic windows skewed towards the SC allele are demarked in blue, while windows skewed towards the SD allele are in red. The dotted lined mark P-value thresholds assuming a z-score of 2, based on the variation observed within the SC lines (blue) and within the SD lines (red). Colored squares on the chromosomes mark genomic regions consistent with: uniform selection (green), mitonuclear incompatibilities favoring coadaptation (blue for SC and red for SD), and mitonuclear antagonistic selection (red lines).
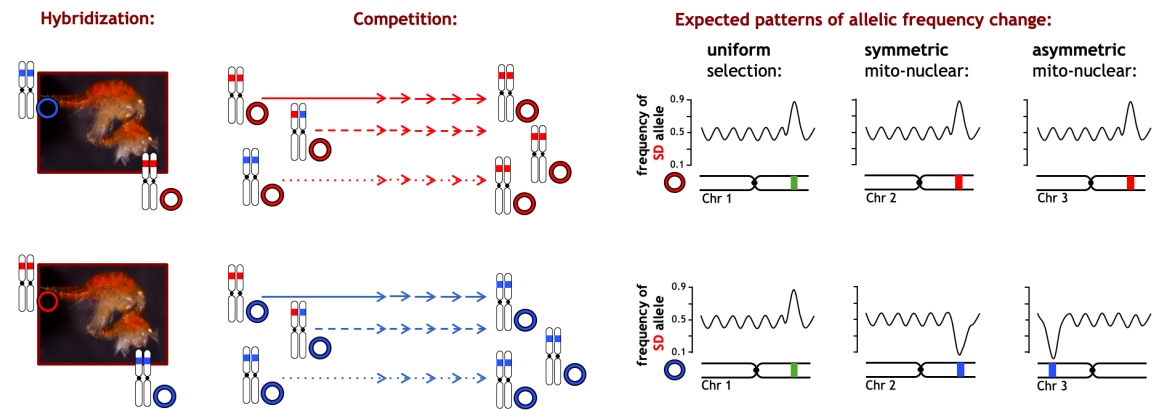


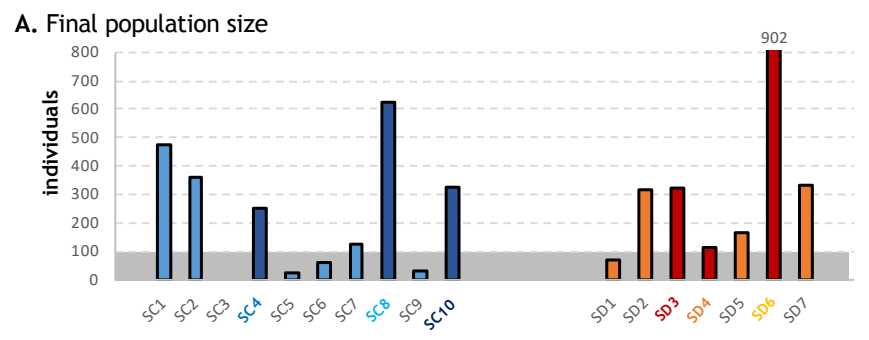

B. Final fitness for fecundity

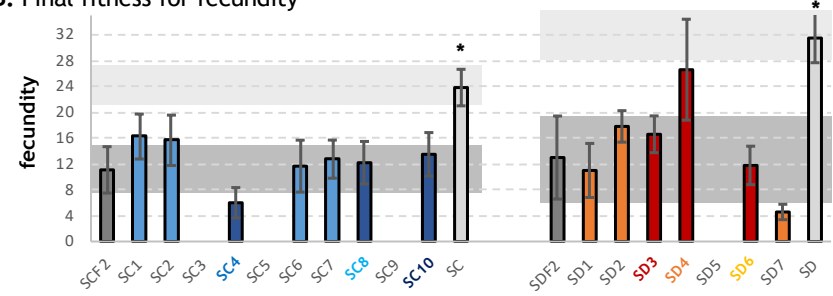

C. Final fitness for survivorship

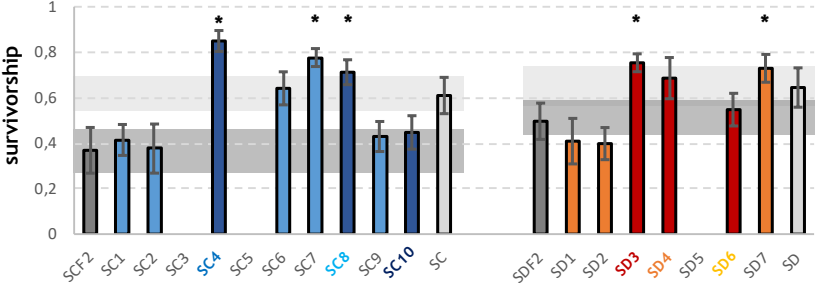

\section{Survivorship over time}
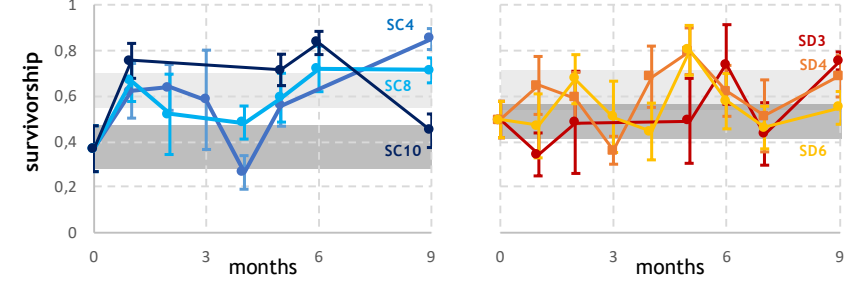


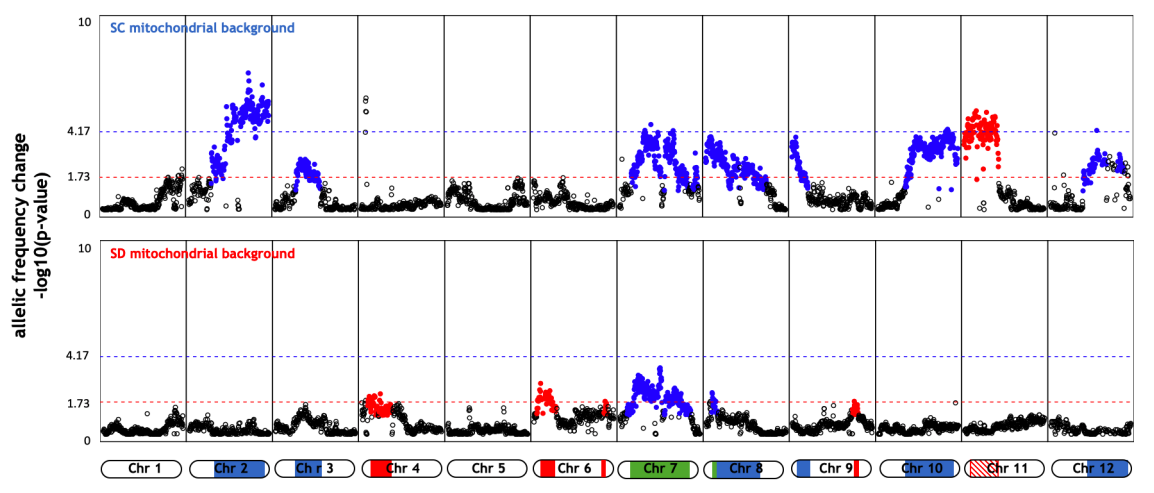

